\title{
Evaluation of the implementation of directly observed treatment short course by private medical practitioners in the management of tuberculosis in Enugu, Nigeria
}

\author{
T.A. OKEKE* \& E.N. AGUWA \\ Department of Community Medicine, University of Nigeria Teaching Hospital, \\ P.M.B. 01129, Enugu, Nigeria
}

\begin{abstract}
This study was carried out to evaluate the use of directly observed treatment short course by private medical practitioners in the management of tuberculosis (TB) as a step towards achieving public-private mix strategy in Enugu, Nigeria. A self-administered questionnaire was used to collect data from all private practitioners practicing in Enugu North Local Government Area. Three hundred and forty doctors were studied and among these $64(18.8 \%)$ managed more than 5 tuberculosis cases every month. Most of the doctors, 310 (91.2\%) based their diagnosis of tuberculosis on sputum acid-fast bacilli result. However, none of the private practitioners used the appropriate drug combination, dosage and duration. Most of the private practitioners $(61.5 \%)$ did not follow up their tuberculosis patients and a larger percentage $(72.1 \%)$ did not have facilities for tracing defaulters. Although $321(94.4 \%)$ of private practitioners would like to be provided with information about national tuberculosis control programme (NTP) only $32(26.2 \%)$ of them had ever participated in a continuing medical education (CME) on tuberculosis. In conclusion, although private practitioners manage a reasonable number of tuberculosis patients their management is often inappropriate and not in line with NTP guidelines. As part of the efforts to achieving a successful public-private mix in TB control, private practitioners should undergo CME on national tuberculosis control programme. It is important that the government should routinely monitor and evaluate the management of TB by the private practitioners.
\end{abstract}

Keywords: Tuberculosis, private practitioners, treatment strategy, Nigeria

\section{Introduction}

In spite of milestones in developing effective antituberculosis drugs, tuberculosis (TB) continues to be a major public health problem all over the world (WHO, 1996). Studies indicate that globally more than 1.7 billion people are infected with TB while 8 million develop the disease. The reasons for this deterioration in increased TB prevalence include improper diagnosis and treatment, poor drug compliance, increased travel and migration, presence of multi-resistant $\mathrm{TB}$ and lately to the pandemic of Human Immunodeficiency Virus (HIV) (WHO, 1989). As an attempt to reduce TB prevalence, WHO advocated the use of directly observed treatment short course (DOTS) strategy (WHO, 1994).

However, despite the adoption of this strategy by 119 countries only $40 \%$ of estimated TB cases are notified worldwide (WHO, 1989). Hence, recently, to improve DOTS implementation WHO has begun addressing the issue of private providers in TB control. Some experts believe that private practitioners not only out number public health care providers in some countries but also manage a large proportion of the unreported TB cases (Lonnroth et al., 2004). Private practitioners also often offer better geographical access and more personalized care than the public facilities (Lonnroth et al., 2004). This resulted in the formation of a global TB control strategy called public-private mix for DOTS implementation (PPM DOTS) (Uplekar, 2003). For PPM DOTS to be effective it may be necessary to identify how much the private practitioners know about TB control and their willingness to be educated on the DOTS strategy. The objective of this study was, therefore, to evaluate the use of DOTS strategy in the management of tuberculosis patients by private medical practitioners in Enugu North Local Government Area (LGA) in Nigeria.

\section{Materials and Methods}

This cross-sectional descriptive study was conducted in 2004 in Enugu North Local Government Area of Enugu State, Nigeria. The only TB treatment centre in this area is the University of Nigeria Teaching Hospital TB unit. The total number of private medical practitioners registered with the Nigerian Medical and Dental Council (NMDC) and practicing in study area was 340 and all these were recruited for the study. Data collection was by self administered questionnaires. 
The knowledge, attitude and practice of private medical practitioners on DOTS strategy in tuberculosis management were explored.
(58.8\%) of the respondents had worked for 615 years as doctors. The majority $(81.2 \%)$ of the private practitioners managed less than 5

Table 1: Diagnostic criteria used by private practitioners in management of tuberculosis

\begin{tabular}{lc}
\hline Diagnostic criteria & Number of private practitioners (\%) \\
\hline Sputum AFB & $312(91.8)$ \\
Chest X-ray & $256(75.3)$ \\
Mantoux & $165(48.5)$ \\
Full blood count / Erythrocyte sedimentation rate & $11(3.2)$ \\
History of contact with a chronically coughing patient & $5(1.5)$ \\
\hline
\end{tabular}

The drug regimen used in TB centres for treating new cases of tuberculosis is that recommended by the National Tuberculosis Control Programme (NTP). It includes two phases namely the intensive phase and the continuation phase. In the intensive phase Rifampicin (R), Isoniazid $(\mathrm{H})$, Pyrazinamide $(Z)$ and Ethambutol are given for two months while in the continuation phase Ethambutol and Isoniazid are given for 6 months (2RHZE/6EH). For the drug dosages, respondents were asked the dose they would give to a man weighing $60 \mathrm{~kg}$. For this the NTP recommends $\mathrm{R}=600 \mathrm{mg}, \quad \mathrm{H}=300 \mathrm{mg}, \mathrm{Z}=1600 \mathrm{mg}, \mathrm{E}=1200 \mathrm{mg}$. Responses of the private medical practitioners were compared to the NTP TB treatment regimen. Data analysis was done based using Statistical Packages for Social Sciences (SPSS). tuberculosis cases every month while 64 (18.8\%) managed more than 5 tuberculosis cases monthly. The most important symptoms suggestive of tuberculosis were persistent productive cough, 276 (81.2\%); haemoptysis $220(64.7 \%)$; chest pain 212 $(62.4 \%)$ and weight loss $209(61.5 \%)$.

Most of the practitioners $(91.2 \%)$ based their diagnosis of tuberculosis on sputum acid-fast bacilli (AFB) result while $256(75.3 \%)$ also did chest radiograph and $165(48.5 \%)$ included Mantoux in their diagnosis. Very few practitioners (3.2\%) included full blood count and erythrocyte sedimentation rates as part of investigation (Table 1 ). The prescription pattern of private practitioners while treating tuberculosis is summarized in Table 2. Only $1.5 \%$ and $2.6 \%$ of the private practitioners used the WHO recommended drug combination

Table 2: Use of short course chemotherapy to treat TB by private practitioners

\begin{tabular}{llc}
\hline Drug & \multicolumn{2}{l}{ Prescription by private practitioners } \\
& Correct $(\%)$ & Wrong (\%) \\
\hline Drug combination (RHZE/EH) & $5(1.5)$ & $335(98.5)$ \\
Drug dosage in an adult weighing 60kg & $9(2.6)$ & $331(97.4)$ \\
Drug duration (2RHZE / 6EH) & $0(0.0)$ & $340(100.0)$ \\
\hline
\end{tabular}

\section{Results}

Three hundred and forty medical practitioners were studied and of these 19(5.6\%) were females while 321 $(94.4 \%$ ) were males (mean age = 33.6years; SD = 7.2970; $\mathrm{df}=339 ;$ 95\% Confidence Interval $=32.849-34.396)$. A larger proportion and dosage, respectively. None of them used the recommended duration of treatment for each drug. The knowledge and implementation of the directly observed treatment short course strategy such as record keeping, surveillance and diagnosis using sputum AFB by private practitioners is summarized in Table 3. Other results showed that

Table 3: Knowledge and implementation of some DOTS strategy by private practitioners

\begin{tabular}{lll}
\hline Variable & Yes (\%) & No (\%) \\
\hline Have you heard about DOTS strategy & $189(55.6)$ & $151(44.4)$ \\
Do you keep records of TB patients & $184(54.1)$ & $156(45.9)$ \\
Do you follow up patients after TB treatment & $131(38.5)$ & $209(61.5)$ \\
Do you have facilities for tracing defaulters & $95(27.9)$ & $245(72.1)$ \\
\hline
\end{tabular}


though most of the private practitioners, 321 $(94.4 \%)$, would like to be provided with information about national tuberculosis control programme only $89(26.2 \%)$ of them had ever participated in a continued medical education programme on TB treatment or control within the past 5 years.

\section{Discussion}

Tuberculosis control is one of the many public health challenges for which innovative approaches to public-private partnership are being sought. This is partly because the private health sector is often the first point of contact for many patients with tuberculosis. Although the present study showed that only a small proportion (18.8\%) of private practitioners managed more than five TB cases per month, studies elsewhere indicate that private practitioners manage reasonably high number of tuberculosis cases. (Uplekar et al., 2001). The later study in India noted that $60 \%$ of individuals with a longstanding cough first went to a private practitioner (Uplekar et al., 2001). Indeed evidence shows that in many low-income countries much of the populations across all socio-economic strata are known to turn to individual or institutional private health care providers (Bennett, 1991). Preference of some tuberculosis patients to private practitioners may be because they are often located close to and are often trusted by the community.

Since a reasonable number of tuberculosis patients are first treated by private practitioners, many agencies like the World Health Organization, have tried scrutinizing the diagnostic criteria and treatment of these cases by the private practitioners with the aim of improving tuberculosis management, hence the establishment of the public-private mix. In fact, all of the published studies from Asia and many other parts of the developing countries have identified important shortcomings in diagnosis of tuberculosis cases by the private practitioners (Uplekar \& Sheperd, 1991; Hong et al., 1995; Marsh et al., 1996). In a previous study, despite the well-established fact that depending just on only chest radiograph for diagnosis of tuberculosis is unreliable due to it's likelihood to show false positive and false negative results yet a larger proportion of private practitioners depended on chest radiograph alone for diagnosis. Fortunately, in present study most private practitioners included sputum AFB result as part of diagnosis. The dependence on sputum AFB in present study may be because of increased awareness of private practitioners on the need for a sputum smear positive result for diagnosis.

Another shortcoming of private practitioners is in treatment of tuberculosis cases using the WHO recommended short course chemotherapy regimen. The present study showed that none of the private practitioners prescribed the drugs for the recommended duration. In a previous study the majority of practitioners treated tuberculosis cases with inappropriate regimen of anti-tuberculosis drugs (Portero \& Rubio, 2003). Recently, private practitioners working in the slums of Mumbai, India were reported to prescribe 80 different drug regimens to their patients with pulmonary tuberculosis and that most of these regimens were inappropriate and expensive (Portero \& Rubio, 2003). This indicate that private practitioners tend to deviate from recommended tuberculosis management practices, which may be due to a gap in knowledge of the DOTS strategies. The fear of ineffective treatment leading to continued transmission of infection and the promotion of multi-drug resistant tuberculosis has led to suggestions on ways to improve the knowledge of private practitioners on DOTS as part of establishing public-private mix strategy.

In our study, private practitioners were found to have other constraints, which must be addressed for them to be usefully integrated to tuberculosis management. For instance, although in the present study over half of the private practitioners have heard about DOTS strategy and keep record of their patients only a few follow up patients after tuberculosis treatment and have facilities for tracing defaulters. These findings are similar to those of many other researchers (Uplekar et al., 2001). In a study by Kumar (2001) about 20\% of private practitioners maintain a record of tuberculosis patients under their treatment while none of them had facility to trace a defaulting patient receiving tuberculosis treatment. The lack of contact tracing facilities among private practitioners in Enugu is most likely to be due to the fact that many of them do not place much importance to contact tracing in TB management.

These findings indicate that the private practitioners do not implement most of the DOTS strategies which include political commitment, case detection through sputum smear microscopy, standardized treatment for 6-8 months, uninterrupted supply of drugs and standardized recording and reporting system. Uninterrupted supply of drugs is an essential DOTS strategy. For this to be achieved the drugs must not only be always available when needed but also free especially in poor income communities where people may not be able to afford the drugs. This study, however, indicate that most private practitioners either provide anti-tuberculosis drugs at a cost or ask patients to buy these drugs (data not shown). 
It has been opined that one of the reasons for not following the recommended NTP regimen by private practitioners may be due to ignorance. Present study observed that though most private practitioners are interested in receiving information about NTP only few of them have ever participated in any continued medical education programme on tuberculosis treatment or control and even fewer have ever been contacted by the health department about NTP. Similar interest in knowing the NTP was also observed in a previous study where $64 \%$ of private practitioners showed interest in receiving information (Uplekar et al., 2001). Hence, lack of information rather than refusal to comply may be the reason for the poor treatment regimen given to tuberculosis patients by private practitioners.

In conclusion, involving private practitioners in management of tuberculosis is vital to achieving tuberculosis control since records show that they first manage a good number of tuberculosis patients. However, the treatment regimen used by private practitioners is inappropriate with poor contact tracing and inability to trace defaulting patients. Despite the willingness of the private practitioners to receive information on NTP only few have had continued medical education programmes on tuberculosis. It is important that the private practitioners undergo continued medical education on National Tuberculosis Control Programme and their practice should be monitored and supervised by the NTP. Moreover, the government should finance publicprivate mix operations including drug cost and cost for staff for supervision, monitoring and evaluation activities.

\section{Acknowledgements}

The authors greatly appreciate the cooperation of the private practitioners in the study area.

\section{References}

Benneth, S. (1991) The mystique of markets: public and private health care in developing countries. Public Health and Policy Departmental Publications. London School of Hygiene and Tropical Medicine 4, 1-24.
Hong, Y.P., Kwon, D.W., Kim, S.J., Chang, S.C., Kang, M.K., Lee, E.P., Moon, H.D., Lew, W.J. (1995) Survey of knowledge, attitudes and practices for tuberculosis among general practitioners. Tubercle and Lung Disease 76,431-435.

Kumar, D. (2001) Planning a Role for Private Practitioners in TB Control: Obstacles and Opportunities. The Communication Initiative. http: / / www.comminit.com/st 2001/sld3395.html: 1-4.

Lonnroth, K., Uplekar. M., Arora, V.K., Juvekar, S., Lan, N.T.N., Mwaniki, D. \& Pathania, V. (2004) Public-private mix for DOTS implementation: what makes it work? Bulletin of the World Health Organization 82, 113.

Marsh, D., Hashim, R., Hassany, F., Hussain, N., Iqbal, Z., Irfanullah, A., Islam, N., Jalisi, F., Janoo, J., Kamal, K., Kara, A., Khan, A., Khan, R., Mirza, O., Mubin, T., Pirzada, F., Rizvi, N., Hussain, A., Ansari, G., Siddiqui, A. \& Luby, S. (1996) Front-line management practices in urban Sindh, Pakistan. Tubercle and Lung Disease 77, 86-92.

Portero, J.L. \& Rubio, M. (2003) Private practitioners and tuberculosis control in the Philippines: strangers when they meet? Tropical Medicine and International Health 8, 329-335.

Uplekar, M. (2003) Involving private health care providers in delivering of TB care: growing strategy. Tuberculosis 83, 156-64.

Uplekar, M.W. \& Shepherd, D.S. (1991) Treatment of tuberculosis by private general practitioners in India. Tubercle 72, 284-290.

Uplekar, M., Pathania, V. \& Raviglione, M. (2001) Private practitioners and public health: weak links in tuberculosis control. Lancet 358, 912916.

WHO (1989) Tuberculosis and AIDS. Bulletin of the International Union Against Tuberculosis and Lung Disease 64, 21-29.

WHO (1994) Framework for Effective Tuberculosis Control. WHO/TB/94.179. World Health Organization, Geneva.

WHO (1996) Group at Risk. WHO's Report on the Tuberculosis Epidemics. World Health Organization, Geneva, 42-55. 